First Nations

\section{pneumonia admissions: Different patients or different attitudes?}

In this issue of the Canadian Respiratory Journal, 1 Marrie et al (pages 336-342) present a database study of hospital admissions among First Nation Aboriginals (FNAs) in Alberta that is fascinating, at least to me. They captured all hospital admissions for "status" FNAs from 1997 to 1999, along with data on where and how long they were hospitalized, the severity of the pneumonia, the number of comorbidities present, whether they were readmitted and the costs involved. They compared these finding with a group of age- and sex-matched non-FNAs who were also hospitalized for pneumonia. There are, of course, weaknesses in the study that are commonly occur in most exercises using administrative databases. Pneumonia is a hospital record diagnosis (there is no information about chest $\mathrm{x}$-rays, sputum cultures, etc). Pneumonia severity assessment relies on information regarding hospital transfers, intensive care unit admissions and events such as shock, artificial ventilation and death (there is no information available to apply an accepted grading system) (1). Further, "status" FNAs were probably not entirely representative of FNAs in general; indeed, some nonstatus FNAs may well have been included in the control group. However, I strongly doubt that these or similar objections are substantial enough to greatly influence the findings of Marrie et al.

The average age of FNA pneumonia patients was just over 53 years, which is surprisingly low. This probably does not mean that pneumonia is not a disease of the elderly among FNAs, but reflects the low average age of the FNA community. In any event, the comparisons of Marrie et al involved an agematched control group. The most striking finding of the study is that the hospital admission rate for the FNAs group was approximately five times that of the control group. Further, pneumonia in FNAs was, on average, less severe than in nonFNAs (ie, fewer intensive care unit admissions and deaths, etc) and hospital stays were approximately $20 \%$ shorter in FNAs. Paradoxically, readmissions were higher in FNAs than in the control group.

What are we to make of this? There are two explanations that are not mutually exclusive: pneumonia may somehow be different in FNAs and non-FNAs; or the health care system may treat FNAs differently from non-FNAs, regardless of the biology of the pneumonia. Marrie et al tried to explore the first of these explanations. They found that the overall rate of comorbidities was similar among FNAs and non-FNAs, though their pattern differed somewhat. FNAs had higher rates of diabetes and substance abuse, while non-FNAs had higher rates of malignancies and vascular disease. It is possible that these patterns accounted for some of the difference in pneumonia
Hospitalisations pour pneumonie chez les Autochtones des Premières nations : attitudes différentes ou patients différents?

ans le présent numéro du Canadian Respiratory Journal, Marrie et son équipe (p. 336-342) font état d'une étude fondée sur une base de données concernant les hospitalisations parmi les Autochtones des Premières nations en Alberta, étude surprenante par ses résultats, tout au moins à mes yeux. Les auteurs ont recueilli toutes les données sur les hospitalisations portant la mention «Indiens inscrits » et datées entre 1997 et 1999, ainsi que les données sur le lieu et la durée d'hospitalisation, la gravité des pneumonies, le nombre de maladies concomitantes, les réadmissions et les coûts. Ils ont comparé ces données à celles d'un groupe de nonAutochtones des Premières nations appariés selon l'âge et le sexe et également hospitalisés pour une pneumonie. Bien sûr, à l'instar des analyses fondées sur des bases de données administratives, l'étude comporte des faiblesses. Le mot «pneumonie » au dossier d'hôpital indique un diagnostic, mais l'on ne dispose d'aucun renseignement sur les radiographies pulmonaires, les cultures d'expectorations, etc. De plus, la gravité des pneumonies ne repose que sur des renseignements indirects comme les mutations dans d'autres hôpitaux, les admissions aux services de soins intensifs, les événements tels qu'un état de choc, la ventilation artificielle ou la mort (rien qui ne puisse permettre l'application d'un système de classification reconnu) (1). De plus, les «Indiens inscrits » étaient probablement non tout à fait représentatifs des Autochtones en général; en effet, certains « Indiens non inscrits » ont pu très bien se retrouver dans le groupe témoin. Cependant, je doute fort que ces observations ou d'autres de même nature puissent changer fondamentalement les résultats de l'étude.

Lâge moyen des patients chez les Autochtones atteints d'une pneumonie dépassait légèrement 53 ans, ce qui est étonnamment bas. Cela ne veut pas dire nécessairement que la pneumonie ne soit pas une maladie de personnes âgées dans cette collectivité, mais le chiffre est un reflet d'un âge moyen bas. De toute façon, Marrie et son équipe ont pris le soin d'apparier un groupe témoin selon l'âge pour établir les comparaisons. Toutefois, l'élément le plus frappant de l'étude est le taux d'hospitalisation chez les Autochtones : il est environ cinq fois supérieur à celui enregistré dans le groupe témoin. De plus, les pneumonies chez les Autochtones étaient en moyenne moins graves que chez les nonAutochtones, si on en juge d'après les admissions aux services de soins intensifs, les décès, etc., et la durée des séjours était à peu près $20 \%$ plus courte. Paradoxalement, le taux de réadmission était plus élevé chez les Autochtones que dans le groupe témoin.

Que faut-il comprendre de tout cela? Il peut y avoir deux explications aux résultats de l'étude et elles ne sont pas mutuellement exclusives : premièrement, les types de pneumonie peuvent différer quelque peu entre les deux groupes; deuxièmement, le système de santé peut traiter les Autochtones différemment des nonAutochtones, indépendamment de la nature de la pneumonie. 
incidence, but almost certainly not all of it. For example, the rate of hospitalization for aspiration pneumonia, a disease associated with substance abuse, was also five times higher among FNAs, but accounted for only a bit more than $1 \%$ of total cases. Further, comorbidities biasing toward admission would also bias toward unfavourable outcomes, which were lower in FNAs than in non-FNAs. Marrie et al believed that their data do not show that FNAs are a more unhealthy population than their controls and it is hard to disagree with them.

The second explanation for the findings of Marrie et al, is that given an FNA patient and a non-FNA patient with comparable cases of pneumonia, the former is more likely to be admitted to hospital than the latter, that is, status per se influences medical decision making. This would account for the lower severity of disease, the shorter hospital stays, and perhaps, even for the higher readmission rates among FNAs. There are indications that this may have been the case. Over $80 \%$ of FNA patients were nonurban dwellers and most were hospitalized in nonurban locales, while only $55 \%$ of the controls were nonurban and they were more likely to be hospitalized in cities. Nonurban FNA patients lived at greater distances from the hospital and were more likely to be hospitalized outside their home region than were nonurban controls. These facts could well have influenced decisions regarding hospital admission. Finally, hospitalization patterns differed between FNAs and non-FNAs, in that the former tended to use a few hospitals (11 of a total of 145 Alberta hospitals accounted for $52 \%$ of FNA pneumonia admissions). Therefore, physician behaviour at a few hospitals could have powerfully influenced the overall result.

Thus, I believe that the high incidence of pneumonia admissions in FNAs is more likely to reflect biases in the health care system than biological differences. Whether these biases are good or bad is another question, as they add expense but may improve outcomes. The issue could be studied further with chart audits, etc, but money would probably be better spent making sure that FNA communities received flu, and perhaps, pneumococcal vaccines, as suggested by Marrie et al.

Nick R Anthonisen MD Editor-in-Chief, Canadian Respiratory Journal

\section{REFERENCE}

1. Ewig S, Ruiz M, Mensa J, et al. Severe community-acquired pneumonia. Assessment of severity criteria. Am J Respir Crit Care Med 1998;158:1102-8.
Marrie et ses coll. ont tenté d'explorer la première hypothèse. Ils ont constaté que le taux général de maladies concomitantes était du même ordre de grandeur dans les deux groupes, bien que les types fussent quelque peu différents. En effet, les Autochtones connaissaient des taux plus élevés de diabète et d'abus d'alcool et de drogues, tandis que les non-Autochtones connaissaient des taux plus élevés de cancers et de maladies cardiovasculaires. Il se peut que ces différences expliquent en partie, mais non en totalité, les écarts de fréquence de la pneumonie. Par exemple, le taux d'hospitalisation pour les pneumonies de déglutition, maladie associée à l'abus d'alcool et de drogues, était également cinq fois plus élevé chez les Autochtones, mais ces pneumonies ne constituaient qu'un peu plus de $1 \%$ de tous les cas examinés. Par ailleurs, les maladies concomitantes qui justifiaient l'hospitalisation auraient dû se traduire par davantage de résultats défavorables, mais c'est le contraire qu'on a observé. Aussi Marrie et son équipe en ont-ils déduit que les données ne laissaient pas transparaître un état de santé général moins bon chez les Autochtones que chez les non-Autochtones, déduction bien difficile à réfuter.

La deuxième explication aux résultats de recherche est que, pour des cas comparables de pneumonie chez un Autochtone et un non-Autochtone, les probabilités d'hospitalisation chez le premier étaient plus élevées que chez le deuxième; autrement dit, le simple fait d'être «Indien inscrit » influait sur les prises de décision médicale. Cela pourrait expliquer le degré moins élevé de gravité des pneumonies, les séjours plus courts et, peut-être même, le taux plus élevé de réadmissions chez les Autochtones, et certains indices portent à croire que c'est bien ce dont il s'agit. En effet, plus de $80 \%$ des Autochtones vivaient en milieu non urbain et la plupart ont été hospitalisés dans des agglomérations rurales, tandis que $55 \%$ seulement des sujets témoins vivaient en milieu non urbain et ils se voyaient plus souvent hospitalisés dans des agglomérations urbaines. Les Autochtones vivant à la campagne demeuraient loin des hôpitaux et ils étaient hospitalisés à l'extérieur de leur région plus souvent que les témoins vivant à la campagne. D'ailleurs, ces faits peuvent très bien avoir joué un rôle dans les décisions d'hospitaliser les malades. Enfin, les types d'hôpitaux différaient également entre les Autochtones et les non-Autochtones en ce sens que les premiers n'avaient recours qu'aux services d'un nombre limité d'hôpitaux (11 hôpitaux sur un total de 145 en Alberta expliquent à eux seuls 52 \% des hospitalisations pour une pneumonie chez les Autochtones). Aussi n'est-il pas impossible que les données de quelques hôpitaux aient pu se répercuter fortement sur l'ensemble des résultats.

Somme toute, je suis porté à croire que la fréquence élevée d'hospitalisations chez les Autochtones pour une pneumonie résulte davantage d'une différence de traitement par le système de santé que de différences biologiques. Quant à savoir si ces écarts sont bons ou mauvais, c'est une autre paire de manches; les hospitalisations font certes augmenter les dépenses, mais elles peuvent aussi donner de meilleurs résultats. On pourrait pousser plus loin l'étude de la question par des revues de dossiers, etc., mais on ferait probablement meilleur usage de l'argent dépensé si on s'assurait que les communautés d'Autochtones étaient vaccinées contre la grippe et, peut-être, contre les maladies à pneumocoques, comme l'ont suggéré Marrie et ses collaborateurs.

Nick R. Anthonisen, MD rédacteur en chef $d u$ Canadian Respiratory Journal

RÉFÉRENCE

1. Ewig S, Ruiz M, Mensa J, et al. Severe community-acquired pneumonia.

Assessment of severity criteria. Am J Respir Crit Care Med 1998;158:1102-8. 


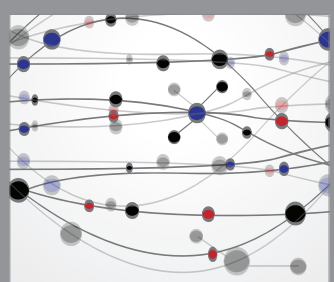

The Scientific World Journal
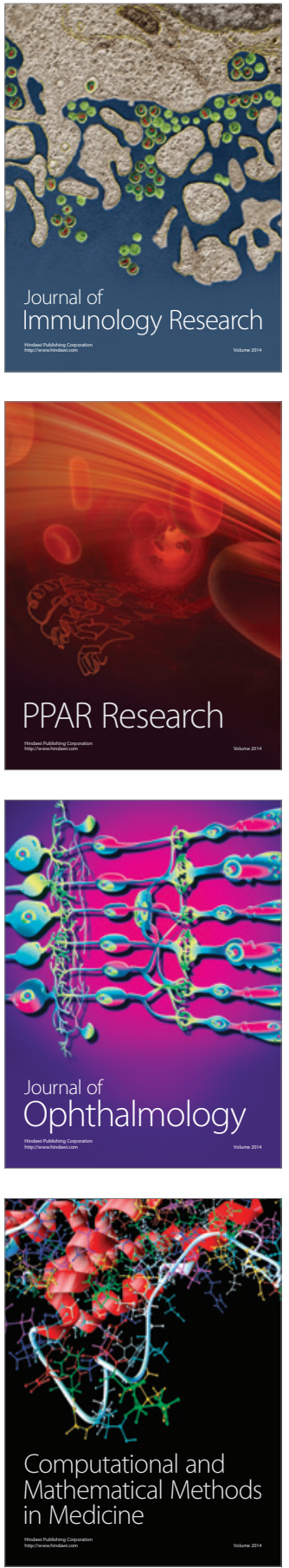

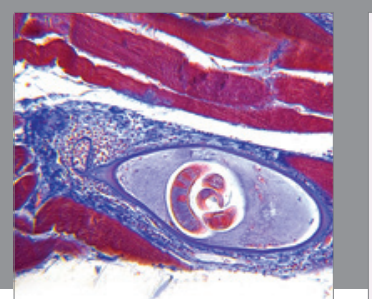

Gastroenterology Research and Practice

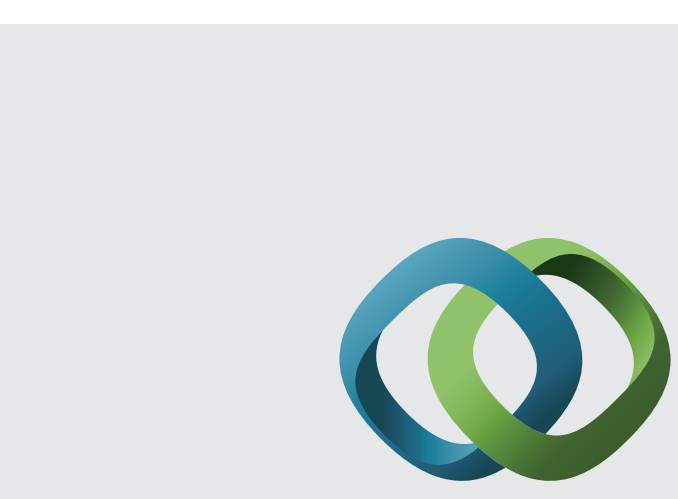

\section{Hindawi}

Submit your manuscripts at

http://www.hindawi.com
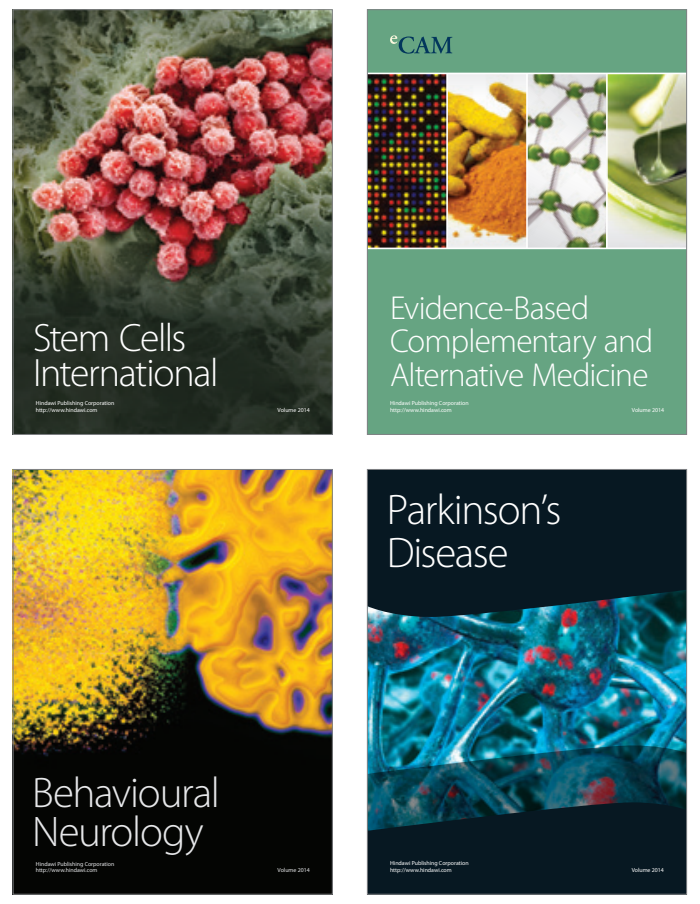
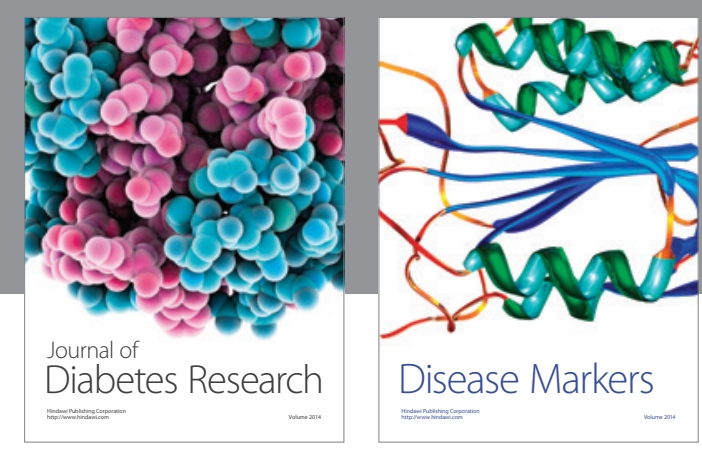

Disease Markers
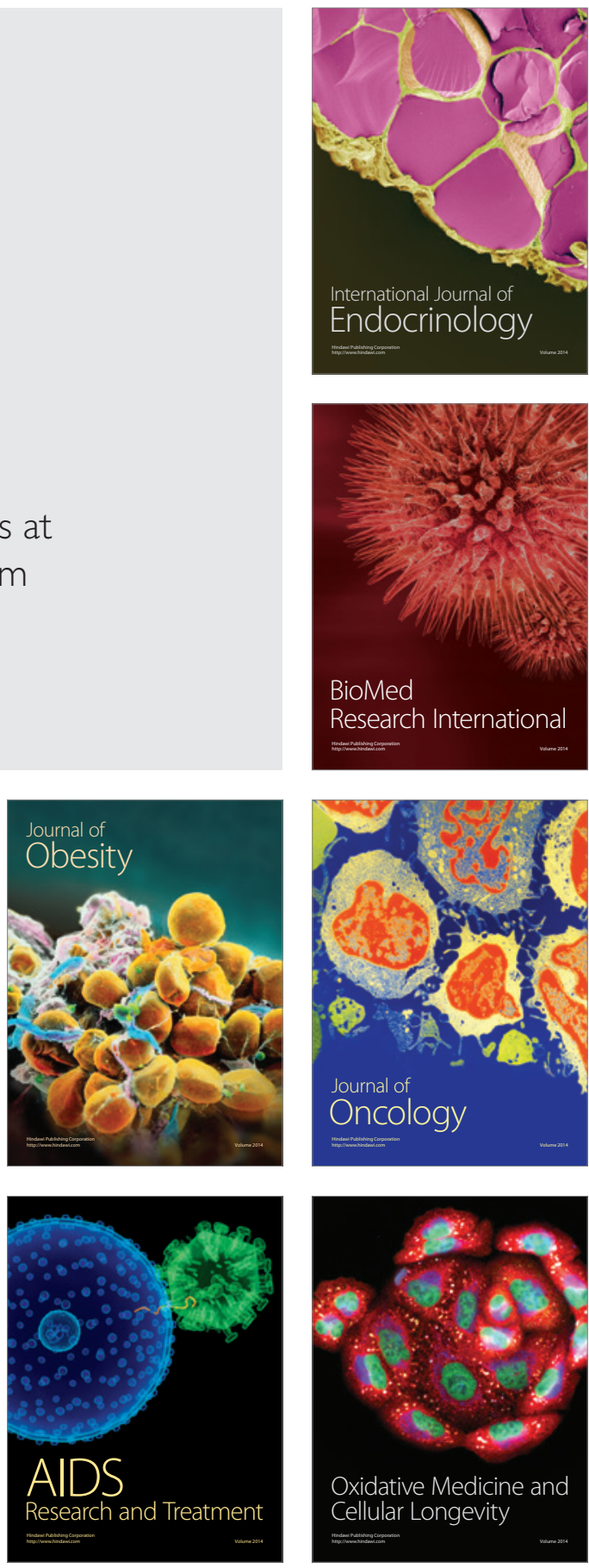